\title{
The perception of transparency with achromatic colors
}

\author{
JACOB BECK \\ University of Oregon, Eugene, Oregon \\ K. PRAZDNY \\ Fairchild Camera and Instrument Corporation, Palo Alto, California \\ and \\ RICHARD IVRY \\ University of Oregon, Eugene, Oregon
}

\begin{abstract}
Metelli has proposed a model of the intensity relationships in perceptual transparency based on Talbot's law of color fusion. Four constraints follow from the application of Talbot's law. Experiments 1 and 2 show that violations of constraints $i$ and ii adversely affect the perception of transparency, while violations of constraints iii and iv do not. Many common occurrences of transparency are in terms of subtractive, rather than additive, color mixture. The constraints derived from the Metelli model are also shown to hold for subtractive color mixture with a filter. An assumption of the Metelli model is that the degree of perceived transparency varies linearly with reflectance. Experiment 3 indicates that the degree of perceived transparency with "partial transparency" varied linearly, not with reflectance, but with lightness, a nonlinear function of reflectance. Experiment 4 indicates that judgments of the degree of transparency with "complete transparency" are based on stimulus relations that differ from those that determine whether the perception of transparency occurs. The results are discussed in terms of how the pattern of intensities relates to other stimulus information, such as figural configuration, in producing the perception of transparency.
\end{abstract}

Metelli (1974a, 1974b) has proposed a model for the intensity relationships in perceptual transparency. The central assumption of Metelli's model is that transparency occurs in accordance with Talbot's law of color fusion. The proximal stimulus resulting when an episcotister rotates in front of surfaces $A$ and $B$ is depicted in Figure 1. Rotating the episcotister rapidly produces the perception of a transparent color (regions $d$ and $c$ ) lying in front of surfaces $A$ and B. According to Talbot's law, the apparent reflectances of regions $d$ and $c$ are equal to

$$
\begin{aligned}
& d=\alpha a+(1-\alpha) e, \\
& c=\alpha b+(1-\alpha) e,
\end{aligned}
$$

where $\alpha$ is the areal fraction occupied by the open sectors of the episcotister, $1-\alpha$ is the areal fraction occupied by the blades of the episcotister, $a$ is the reflectance of surface $A, b$ is the reflectance of surface

This research was supported by NSF Grant MCS-79-23422 to the Computer Vision Laboratory, University of Maryland, and by AFOSR Contract F49620-83-C-0093 to the Psychology and Computer and Information Sciences Departments, University of Oregon. J. Beck's mailing address is: Department of Psychology, University of Oregon, Eugene, OR 97403.
A $B$

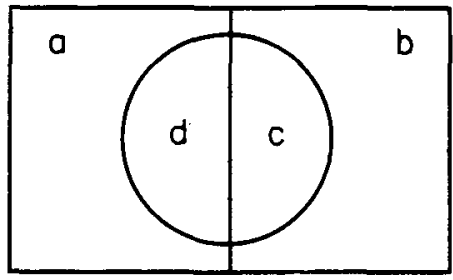

Figure 1. The proximal stimulus resulting from an episcotister rotating in front of two surfaces differing in reflectance. Capital letters $A$ and $B$ indicate the background surfaces. Lowercase letters indicate regions of differing intensity.

$B$, and $e$ is the reflectance of the episcotister blades. Solving Equations 1 and 2 for $\alpha$ and e yields

$$
\begin{gathered}
\alpha=(d-c) /(a-b) \\
e=(a c-b d) /(a+c)-(b+d) .
\end{gathered}
$$

Alpha is the proportion of the apparent reflectances of $d$ and $c$ determined by the reflectances a and $b$ and is an index of the transparency of the apparent disk. Since $\alpha$ is restricted to values between 0 and 1 , Equation 3 implies that (i) if $a>b$, then $d>c$, 
and vice versa, if $a<b$, and (ii) the absolute difference $|a-b|$ must be greater than the absolute difference $|d-c|$. Constraint $i$ is a restriction on the order of the intensities and insures that $a$ is positive. Constraint ii is a restriction on the magnitudes of the intensities and insures that $a$ is less than 1 . Since $e$ is also restricted to values greater than or equal to 0 and less than or equal to 1 , order and magnitude constraints can also be derived from Equation $4 .^{1}$ Equation 4 implies that (iii) if $(a+c)\rangle(b+d)$ then $a c\rangle$ $b d$, and vice versa, if $(a+c)<(b+d)$, and (iv) the $a b-$ solute difference $|(a+c)-(b+d)|$ must be equal to or greater than the absolute difference $|a c-b d|$. Constraint iii insures that $e$ is nonnegative, and constraint iv insures that $e$ is less than or equal to 1 . The four constraints are independent. Numerical values can be assigned to the reflectances $a, b, c$, and $d$ in Equations 3 and 4 that satisfy three of the constraints but not the fourth.

Metelli (1974b) has demonstrated that the perception of transparency occurs when constraints $i$ and ii derived from Equation 3 are met and fails to occur when either of these constraints are violated. He has, however, not investigated the consequences of violating constraints iii and iv derived from Equation 4. This may be because it does not seem that they would affect the perception of transparency, since it appears doubtful that people are able to make the judgments required by constraints iii and iv. To anticipate, we will present evidence that violations of constraints iii and iv do not adversely affect the perception of transparency. We argue that the computations carried out by the visual system in perceiving transparency are in terms of lightness values rather than in terms of reflectances or luminances. Processing of the intensity information involves checking whether the lightnesses in a pattern satisfy the order restrictions of constraint $i$ and the magnitude restrictions of constraint ii. Constraints iii and iv involve operations of addition and multiplication that are not readily interpretable in terms of lightness values.

The present study seeks to clarify both the factual background and the theoretical issues in the perception of transparency. Six experiments test and extend the Metelli model. A model of the intensity relationships when transparency occurs in terms of a filter that transmits light is also presented. The relations between the intensity values when transparency occurs with a filter and when it occurs with an episcotister are compared.

\section{EXPERIMENT 1}

Experiment 1 was designed to investigate how the pattern of image intensities and the figural configuration affected the perception of transparency. Two supplementary experiments answering questions raised by Experiment 1 are also reported.

\section{Method}

Stimuli. A PDP-11 computer was used to generate two sets of Polaroid pictures at the Computer Vision Laboratory, University of Maryland. A computer controlled a flying spot in focus on a CRT, and an oscilloscope camera imaged the CRT face plate onto the film. The stimuli in Set 1 consisted of four regions differing in reflectance and depicted two overlapping surfaces, $B$ and $D$, on a larger background surface, $A$ (Figure 2a). Capital letters in the figures identify depicted surfaces and lowercase letters regions of differing reflectance. The figures $B$ and $D$ were $2.7 \times 2.4 \mathrm{~cm}$, and the area of overlap was $1.3 \times 1.2 \mathrm{~cm}$. The background figure $A$ was $6.6 \times 6.0 \mathrm{~cm}$. The four reflectances were programmed to differ by equal increments. The reflectances of 10 stimuli were measured with a Spectra-Pritchard photometer. The mean reflectances of the four regions were $.22(\mathrm{SD}=.02), .34(\mathrm{SD}=.02), .47(\mathrm{SD}=.02)$, and $.59(\mathrm{SD}=.03)$.

Set 1 contained 24 stimuli corresponding to the 24 possible permutations of the four different reflectances. A stimulus is identified by a sequence of the four letters, a, b, c, and d, for example, dcba. The letter order indicates increasing gray levels from lowest to highest. Table 1 lists the 24 stimuli. Stimuli 1 through 10 satisfied or effectively satisfied constraints i and ii. (Stimuli 1, 2, 4, 5, and 7 through 10 technically violated constraint ii since the absolute difference $|c-d|$ was equal to the absolute difference $|a-b|$. An $a$ equal to 1 is the limiting value for the occurrence of transparency. Observations, however, indicated that the perception of transparency is affected only if constraints $i$ and ii are clearly violated. Stimuli that violated constraints at limiting values will be considered effectively to satisfy the constraints.) Stimuli 11 through 16 violated constraint $i$ in a strong sense. If the gray levels in the four quadrants at the $x$-junction in the upper left of Figure $2 a$ are traced out in increasing magnitude, the gray levels crisscross (Figure 2b). Stimuli 17 and 18 violated constraint ii in the strong sense that the gray level interval ab is contained within the gray level interval cd. Stimuli 19 and 20 violated both constraint $i$ and constraint ii strongly. Stimuli 21 through 24 violated constraint $i$, but the gray levels do not crisscross. They effectively satisfied constraint ii.

Since $(a+c)-(b+d)$ is equal to 0 for stimuli $1,2,4,5$, and 7 through $10, e$ is undefined and constraints iii and iv are not satisfied. On each of the stimuli decreasing the highest reflectance by $4 \%$ satisfied constraints iii and iv. Stimuli $1,2,4,5$, and 7 through 10 may be considered to weakly violate constraints iii and iv. The remaining stimuli in Set 1 satisfied both constraint iii and constraint iv.

Set 2 investigated the effect of figural configuration on the perception of transparency. Configuration 1 was similar to that in Set 1 and is illustrated in Figure 38. The overlapping figures $B$ and $D$ were a square $2.6 \mathrm{~cm}$ on a side and a rectangle $4 \times 2 \mathrm{~cm}$. The area
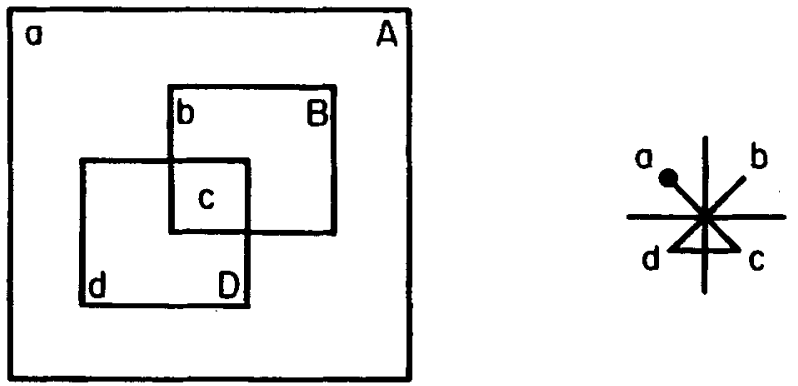

(a)

(b)

Figure 2. (a) Stimulus configantion in Set 1. Capital letters indicate the aurfaces depleted. Lowercase letters indicate regions of differing Intensity. (b) Diagram llustrating the criascrosing of gray levels with stimulus acdb (see text). 


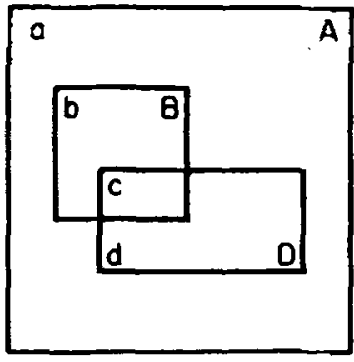

(a)

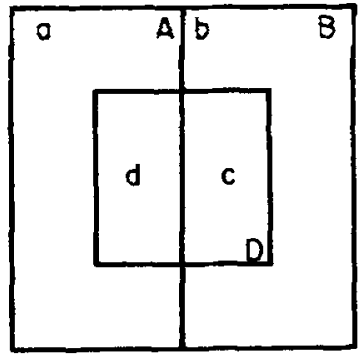

(b) Set 1, permutations of the sume four reflectances, but varied. Table 2 lists the stimulus reflectances. Negative values for $a$ and for $e$ indicate that a stimulus violated constraints $i$ and iii, respectively; absolute values greater than 1 for $\alpha$ and for $e$ indicate that a stimulus violated constraints $i i$ and $i v$.

Set 2 contained $\mathbf{4 0}$ stimuli. Sixteen pairs of stimuli were generated in which corresponding regions in configurations I and II were the same reflectances. Seven stimuli, each with configurations I and II, satisfied constraints $i$ and ii (stimuli 1 through 7 in Table 2). Four stimuli, each with configurations I and II, were generated with the order cdab (stimuli 8 through 11). The order cdab violated constraint $i$. Three stimulus pairs also violated constraint ii. Five stimuli, each with configurations I and II, were generated with the order cdba (stimuli 12 through 16). These pairs of stimuli violated constraint ii but not constraint $i$. Eight stimuli were generated only with configuration I. They violated constraint i strongly and involved a crisscrossing of gray levels (stimuli 17 through 24). These eight stimuli satisfied constraint ii.

Stimuli 4, 5, and 6 in Table 2 violated constraint iii. These violations, however, are small. Increasing the reflectance of $\mathrm{c}$ in stimulus 4 by $2 \%$, in stimulus 5 by $1 \%$, and in stimulus 6 by $2 \%$ satisfied constraint iii. The remaining stimuli in Set 2 satisfied both constraint iii and constraint iv. Sample stimulus displays are shown in Figure 4. It should be noted that the halftone process fails to accurately reproduce the gray values of the stimuli in Figures 4 and 7 .

Procedure. Sets 1 and 2 were alternated. Before presentation of each set of stimuli, subjects were shown examples of overlap with and without transparency. Before presentation of Set 1 , transparency was illustrated by showing the subjects a Polaroid filter overlying gray papers arranged as in Figure 2a. Overlapping without transparency was illustrated by superimposing four gray papers corresponding to regions $a, b, c$, and $d$ in Figure 2a. They appeared opaque. The subjects were told that they would be shown photographs of surfaces arranged as in Figure 28 and asked to judge whether the bottom square-like figure (D) was transparent. They were instructed that they were to report $D$ transparent only if both the background (A) and the top square (B) were seen through $D$. If only $A$ or $B$ was seen through $D$, but not both, the stimulus was to be judged as not transparent. Similar instructions and examples were given before Set 2 was presented. The subjects were again cautioned that a stimulus was to be reported as transparent only if they could see both the top square and the background through the bottom rectangle (Figure 3a) or if they could see through the inner square to both backgrounds rectangles (Figure $3 \mathrm{~b})$. The instructions with both sets also stressed that a stim-

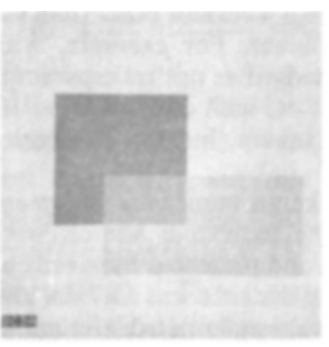

a

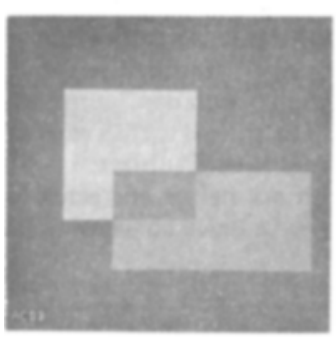

C

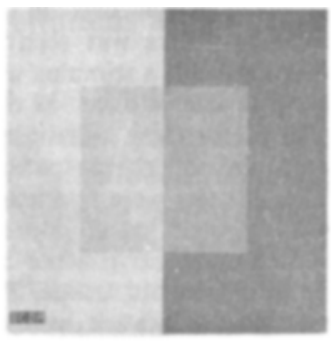

b

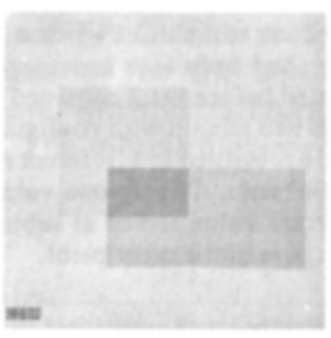

d

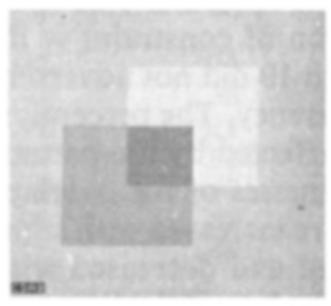

e

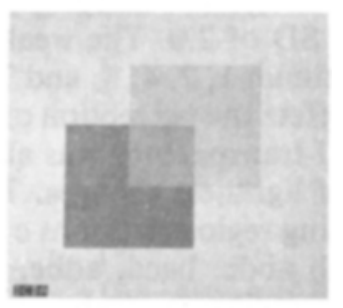

$f$

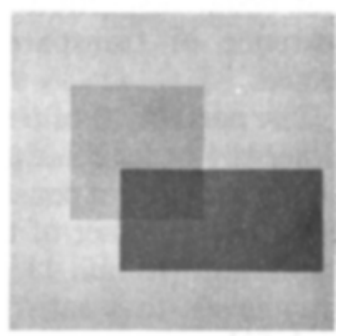

h
Figure 4. Sample stlmull: (a) Confganntion I stimulus satiafyIng constralnts I through IV. (b) Configantion II utmulus setiofyIng comstralnts i through iv. (c) Criacroms atimulus violating constraint i. (d) Stimulus order cdba volating constraint if. (e) Stimulus order cdab evoled trenperency judgments withongh it violates comotnint 1. (f) Stimulae order debe volate constralat if the bottom square to eeen to overite the top square. If the top square is seen to overlie the bottom square, the stimulus order becomes bcda, which satisfies canstrulnts I through iv (see text). (g) Stimulus order cbda in which $\mathrm{c}$ is closer in lightness to b than to $d$. There is a tendency to see the square overtle the rectangle. Both stimulus order cdba (when the square is seen to overtie the rectangle) and cbda satisfy constraines i through (v. (h) Stimalus order cdba in which $\mathrm{c}$ is closer in lightness to $d$ then to $b$. There is a tendency to see the rectangle overlle the square. Both stimulus order edba and stimulus order cbda (when the square is seen to overlic the rectangle) satisfy constraints 1 through iv. 
ulus was to be judged as not transparent if a surface other than the indicated surface was seen as transparent. For example, with configuration I, a stimulus was to be judged as not transparent if the top square was seen as transparent or, with configuration II, if the left or right half of the inner square, but not the entire square, was seen as transparent.

The subjects were instructed to make an immediate judgment based on their visual impression. The individual stimuli were mounted on pieces of white cardboard and presented by means of a Gerbrands tachistoscope. The viewing distance was $59.7 \mathrm{~cm}$ and the exposure duration was 2 sec. A subject initiated a stimulus presentation by pressing a switch. Before presentation of each stimulus set, five pratice stimuli were presented. The five practice stimuli contained two stimuli that, in pretests, had been judged as transparent, two stimuli that had been judged as not transparent, and one stimulus in which a surface other than the indicated surface had been seen as transparent. The five practice stimuli presented before Set 2 consisted of three stimuli with configuration I and two stimuli with configuration II. The stimuli within each set were presented in a different random order to each subject.

Subjects. Twenty-one volunteers with normal or corrected-tonormal vision served as subjects. They were naive concerning the purpose of the experiment.

\section{Results}

Table 1 presents the results with Set 1 . The mean number of transparency judgments of stimuli 1 through 10 satisfying constraints $i$ and ii is 18.5 with a SD of 2.0. The weak violation of constraint iv by stimuli $1,2,4,5$, and 7 through 10 did not adversely affect the perception of transparency. The perception of transparency was also not affected by the pattern of lightness changes. The lightnesses of the overlapping regions (regions $c$ and d) are increased with stimuli abdc, bacd, adbc, and bcad and decreased with stimuli cdba, dcab, cdba, and dacb, and the lightness of the darker surface is increased while the lightness of the lighter surface is decreased with stimuli adcb and bcda. Inspection of Table 1 shows that the occurrence of transparency judgments was not affected.

The perception of transparency did not occur when either the order relations in constraint $i$ or the magnitude relations in constraint ii were violated strongly. The mean number of transparency judgments of the six stimuli (stimuli 11 through 16) with crisscrossing gray levels that satisfied constraint ii but strongly violated constraint $i$ is .67 with a SD of .82 . The mean number of transparency judgments of the two stimuli (stimuli 17 and 18) that satisfied constraint $i$ but strongly violated constraint ii is .50 with a SD of .71. The two stimuli (stimuli 19 and 20) that failed to satisfy both constraint $i$ and constraint ii were never judged to be transparent.

Stimuli 21 through 24 fail to satisfy constraint i. Significant numbers of transparency judgments were obtained with stimuli cdab and badc. The stimulus cdab (Figure 4e) was judged transparent 13 times (over 50\%), and the stimulus badc 6 times (over 25\%). Why do these two stimuli and not the stimuli abcd and dcba produce exceptions? A stimulus is am-
Table 1

Frequency of Transparency Judgments in Set 1

\begin{tabular}{|c|c|c|}
\hline Stimulus No. & Stimulus & Frequency \\
\hline \multicolumn{3}{|c|}{ Satisfied Constraints i and ii } \\
\hline 1. & abdc & 15 \\
\hline 2. & adbc & 18 \\
\hline 3. & adcb & 18 \\
\hline 4. & bacd & 18 \\
\hline 5. & bcad & 21 \\
\hline 6. & bcda & 21 \\
\hline 7. & cbda & 21 \\
\hline 8. & cdba & 18 \\
\hline 9. & dacb & 18 \\
\hline 10. & dcab & 17 \\
\hline Mean & & 18.5 \\
\hline SD & & 2.0 \\
\hline \multicolumn{3}{|c|}{ Failed Constraint i Strongly (Crisscross Stimuli) } \\
\hline 11. & acbd & 1 \\
\hline 12. & $\mathrm{acdb}$ & 2 \\
\hline 13. & bdac & 0 \\
\hline 14. & bdca & 0 \\
\hline 15. & $c a d b$ & 0 \\
\hline 16. & dbca & 1 \\
\hline Mean & & .67 \\
\hline SD & & .82 \\
\hline
\end{tabular}

Failed Constraint ii Strongly (Interval ab is Included Within the Interval cd)

\begin{tabular}{rcc}
17. & cbad & 0 \\
18. & dabc & 1 \\
$\begin{array}{c}\text { Mean } \\
\text { SD }\end{array}$ & & .50 \\
& & .71 \\
19. & Failed Constraints i and ii Strongly \\
20. & cabd & \\
& dbac & 0 \\
21. & Failed Constraint i & 0 \\
22. & abcd & 3 \\
23. & badc & 6 \\
24. & cdab & 13 \\
Mean & dcba & 2 \\
SD & & 6.0 \\
\hline
\end{tabular}

Note-Since $(a+c)-(b+d)$ equals 0 for stimuli $1,2,4,5$, and 7 through $10, e$ is undefined and constraints iii and iv are not satisfied. The remaining stimuli satisfy both constraints iii and iv.

biguous as to whether surface $D$ is seen as overlying surface $B$ or surface $B$ is seen as overlying surface $D$. The theoretical derivation assumes that the overlying transparent regions are $d$ and $c$ and the underlying opaque regions are $a$ and $b$. If surface $B$ is seen as overlying surface $D$, then the regions $b$ and $d$ are interchanged. Thus, the stimulus abcd becomes adbc and the stimulus dcba (Figure 4f) becomes beda. Both adcb and beda satisfied constraints $i$ and ii. What is suggested is that subjects tended to see these surfaces as transparent, with surface B overlying surface $D$. The low number of transparency judgments reflect that the instructions asked subjects to report the stimulus as transparent only if surface $D$ was seen as overlying surface B. Support for this conjecture 
comes from a preliminary study in which the stimuli in Set 1 were presented on slides to a group of 18 subjects. The instructions were similar to those given in Experiment 1. The main difference was that the subjects were first asked to judge whether they saw a stimulus as transparent, and then asked to judge whether surface $D$ was seen to be overlying surface $B$ or whether surface B was seen to be overlying surface $D$. Two subjects judged the stimulus abcd to be transparent with surface D overlying surface B, whereas 16 subjects judged the stimulus to be transparent with surface B overlying surface D (stimulus adcb). Two subjects judged the stimulus dcba to be transparent with surface D overlying surface $B$, whereas 15 subjects judged the stimulus to be transparent with surface B overlying surface D (stimulus bcda). One subject judged the stimulus as not transparent.

In contrast, in stimuli cdab and badc surface B cannot be seen as overlying surface $D$. If surface $B$ is seen overlying surface $D$, then cdab becomes cbad and badc becomes dabc. Both cbad and badc strongly violate constraint ii. The interval cd is included in the interval $a b$. The occurrence of transparency judgments with stimuli cdab and badc indicates that if figural conditions strongly suggest transparency, the perception of transparency occurs even when the pattern of image intensities contradicts it.

Table 2 presents the results of Set 2. The mean number of transparency judgments for the seven stimuli satisfying constraints $i$ and ii with configuration I is 17.9 with a SD of 1.6. The mean number of

Table 2

Stimulus Reflectances and Frequency of Transparency Judgments in Set 2

\begin{tabular}{|c|c|c|c|c|c|c|c|c|c|}
\hline \multirow[b]{2}{*}{ Stimulus No. } & \multirow[b]{2}{*}{ Stimulus } & \multicolumn{6}{|c|}{ Reflectances } & \multicolumn{2}{|c|}{ Frequency } \\
\hline & & a & b & c & d & $a$ & e & $\begin{array}{c}\text { Configura- } \\
\text { tion I }\end{array}$ & $\begin{array}{c}\text { Configura- } \\
\text { tion II } \\
\end{array}$ \\
\hline \multicolumn{10}{|c|}{ Satisfied Constraints $\mathrm{i}$ and $\mathrm{ii}$} \\
\hline $\begin{array}{l}1 . \\
2 . \\
3 . \\
4 . \\
5 . \\
6 . \\
7 .\end{array}$ & $\begin{array}{l}\text { abdc } \\
\text { bcda } \\
\text { bcda } \\
\text { cdba } \\
\text { cdba } \\
\text { cdba } \\
\text { dcab }\end{array}$ & $\begin{array}{l}.09 \\
.54 \\
.54 \\
.56 \\
.55 \\
.41 \\
.19\end{array}$ & $\begin{array}{l}.23 \\
.20 \\
.20 \\
.43 \\
.46 \\
.33 \\
.32\end{array}$ & $\begin{array}{l}.40 \\
.34 \\
.42 \\
.30 \\
.26 \\
.19 \\
.17\end{array}$ & $\begin{array}{l}.30 \\
.47 \\
.47 \\
.41 \\
.32 \\
.26 \\
.11\end{array}$ & $\begin{array}{l}.71 \\
.38 \\
.15 \\
.85 \\
.67 \\
.88 \\
.46\end{array}$ & $\begin{array}{r}.83 \\
.43 \\
.46 \\
-.42 \\
-.14 \\
-.79 \\
.04\end{array}$ & $\begin{array}{l}17 \\
20 \\
20 \\
16 \\
17 \\
18 \\
17\end{array}$ & $\begin{array}{l}15 \\
20 \\
15 \\
14 \\
11 \\
13 \\
16\end{array}$ \\
\hline 7. & & & & & & & & $\begin{aligned} \text { Mean } & =17.9 \\
S D & =1.6\end{aligned}$ & $\begin{aligned} \text { Mean } & =14.9 \\
\mathrm{SD} & =2.8\end{aligned}$ \\
\hline \multicolumn{10}{|c|}{ cdab Stimuli } \\
\hline $\begin{array}{r}8 . \\
9 . \\
10 \\
11\end{array}$ & $\begin{array}{l}\text { cdab } \\
\text { cdab } \\
\text { cdab } \\
\text { cdab }\end{array}$ & $\begin{array}{l}.51 \\
.41 \\
.45 \\
.45\end{array}$ & $\begin{array}{l}.55 \\
.48 \\
.52 \\
.50\end{array}$ & $\begin{array}{l}.17 \\
.20 \\
.09 \\
.28\end{array}$ & $\begin{array}{l}.27 \\
.25 \\
.17 \\
.37\end{array}$ & $\begin{array}{r}-2.50 \\
-.71 \\
-1.14 \\
-1.80\end{array}$ & $\begin{array}{l}.44 \\
.32 \\
.32 \\
.42\end{array}$ & $\begin{array}{r}7 \\
12 \\
9 \\
12\end{array}$ & $\begin{array}{l}2 \\
2 \\
2 \\
0\end{array}$ \\
\hline 11. & & & & & & & & $\begin{aligned} \text { Mean } & =10.00 \\
S D & =2.5\end{aligned}$ & $\begin{aligned} \text { Mean } & =1.5 \\
\mathrm{SD} & =1.0\end{aligned}$ \\
\hline \multicolumn{10}{|c|}{ cdba Stimuli } \\
\hline $\begin{array}{l}12 . \\
13 . \\
14 . \\
15 . \\
16 .\end{array}$ & $\begin{array}{l}\text { cdba } \\
\text { cdba } \\
\text { cdba } \\
\text { cdba } \\
\text { cdba }\end{array}$ & $\begin{array}{l}.52 \\
.43 \\
.52 \\
.52 \\
.57\end{array}$ & $\begin{array}{l}.49 \\
.38 \\
.43 \\
.45 \\
.48\end{array}$ & $\begin{array}{l}.40 \\
.20 \\
.17 \\
.23 \\
.27\end{array}$ & $\begin{array}{l}.47 \\
.34 \\
.36 \\
.42 \\
.41\end{array}$ & $\begin{array}{l}2.33 \\
2.80 \\
2.11 \\
2.71 \\
1.56\end{array}$ & $\begin{array}{l}.56 \\
.48 \\
.66 \\
.58 \\
.86\end{array}$ & $\begin{array}{r}14 \\
12 \\
14 \\
9 \\
17\end{array}$ & $\begin{array}{r}14 \\
11 \\
8 \\
12 \\
14\end{array}$ \\
\hline 16. & & & & & & & & $\begin{aligned} \text { Mean } & =13.2 \\
\mathrm{SD} & =3.0\end{aligned}$ & $\begin{aligned} \text { Mean } & =11.8 \\
S D & =2.5\end{aligned}$ \\
\hline \multicolumn{10}{|c|}{ Crisscross Stimuli } \\
\hline $\begin{array}{l}17 . \\
18 . \\
19 \\
20 \\
21 \\
22 \\
23 \\
24\end{array}$ & $\begin{array}{l}\text { acdb } \\
\text { acdb } \\
\text { bdac } \\
\text { bdca } \\
\text { bdca } \\
\text { bdca } \\
\text { cadb } \\
\text { cadb }\end{array}$ & $\begin{array}{l}.27 \\
.10 \\
.34 \\
.50 \\
.51 \\
.46 \\
.21 \\
.16\end{array}$ & $\begin{array}{l}.44 \\
.36 \\
.24 \\
.23 \\
.23 \\
.22 \\
.38 \\
.32\end{array}$ & $\begin{array}{l}.36 \\
.18 \\
.41 \\
.35 \\
.50 \\
.36 \\
.19 \\
.14\end{array}$ & $\begin{array}{l}.37 \\
.23 \\
.31 \\
.32 \\
.31 \\
.31 \\
.25 \\
.20\end{array}$ & $\begin{array}{r}-.06 \\
-.19 \\
-1.00 \\
-.11 \\
-.68 \\
-.21 \\
-.35 \\
-.38\end{array}$ & $\begin{array}{l}.36 \\
.21 \\
.33 \\
.34 \\
.39 \\
.34 \\
.24 \\
.19\end{array}$ & $\begin{array}{l}8 \\
0 \\
0 \\
1 \\
0 \\
2 \\
5 \\
1\end{array}$ & \\
\hline 24. & & & & & & & & $\begin{aligned} \text { Mean } & =2.1 \\
S D & =2.9\end{aligned}$ & \\
\hline
\end{tabular}

Note-The values of $a$ and $e$ are given by Equations 3 and 4. A negative value for a indicates that a stimulus violates constraint $i$ and for $e$ that it violates constraint iii. An absolute value greater than 1 for a indicates that a stimulus violates constraint if and for $e$ that it violates constraint iv. 
transparency judgments for the corresponding stimuli with configuration II is 14.9 with a SD of 2.8. A $t$ test of the difference between means is significant $[\mathrm{t}(6)=3.44, \mathrm{p}<.05]$. Combining the number of transparency judgments with configurations I and II, the mean number of transparency judgments of the eight stimuli satisfying constraint iii was 17.5 with a SD of 2.2. The mean number of transparency judgments of the six stimuli violating constraint iii was 14.8 with a SD of 2.6. A t test of the difference just misses significance $[t(12)=2.06, p>.05]$.

The four stimuli with the order cdab violated the order relation in constraint $i$. The mean number of transparency judgments with configuration $I$ is 10.0 with a SD of 2.5, and that with configuration II is 1.5 with a SD of 1.0. A t test of the difference is significant $[t(3)=5.47, p<.05]$. Thus, exceptions to constraint $\mathrm{i}$ with stimulus cdab occurred more readily with configuration I than with configuration II. The local cues for transparency are similar for configurations I and II. The $\mathrm{x}$-junctions indicate the possibility of transparency on both configurations. The fact that configuration I was more effective than configuration II in producing the perception of transparency indicates that the global figural configuation affects the perception of transparency. The component regions in configuration II are more regular and symmetric with a nontransparent organization than the corresponding regions in configuration $I$.

The five stimuli with the order cdba satisfied constraint $i$ but failed to satisfy constraint ii. The mean number of transparency judgments with configuration I is 13.2 with a SD of 3.0 and with configuration II, 11.8 with a SD of 2.5. A $t$ test of the difference is not significant $[\mathrm{t}(4)=.93, \mathrm{p}>.4]$.

The eight crisscross stimuli occurred only with configuration $\mathrm{I}$. The mean number of transparency judgments is 2.1 with a SD of 2.9. A relatively large number of transparency judgments (8) ocurred with one of the acdb stimuli (stimulus 17 in Table 2). Region $\mathrm{c}$ in this stimulus differs by $1 \%$ from region $\mathrm{d}^{2}{ }^{2}$ The closeness in lightness of the two regions is likely to have facilitated the perception of transparency. If regions $\mathrm{c}$ and $\mathrm{d}$ are interchanged, the order becomes adcb, which satisfies both the order and magnitude constraints. The results again indicate that if figural cues strongly suggest transparency, then contradictory indications from the pattern of intensities may be overridden.

\section{Supplementary Experiments}

Supplementary Experiment 1. The aims of the experiment were (1) to examine how the magnitude of the violation of constraint $i$ and of constraint ii affects the perception of transparency, and (2) to determine whether the judgments of transparency evoked by stimulus 17 in Experiment 1 can be replicated.
The procedure was the same as in Experiment 1 . Nineteen stimuli were presented. The stimulus arrangement was that of configuration I. Table 3 lists the stimulus reflectances. Nine stimuli satisfied constraints i through iv (stimuli 1 through 9 in Table 3). Four stimuli which violated constraint $i$ were generated (stimuli 10 through 13). The stimulus order was cdab. The reflectance of region $d$ exceeded that of region $c$ by $1 \%, 2 \%, 4 \%$, and $8 \%$. If $c$ and $d$ are permuted, the stimulus order becomes cdab, which satisfies constraint $i$. Two stimuli violated constraint ii (stimuli 14 and 15). The difference in reflectance between regions $d$ and $c$ exceeded that between regions $a$ and $b$ by $4 \%$ for stimulus 14 and $27 \%$ for stimulus 15 . Four crisscross stimuli were presented (stimuli 16 through 19). Twenty-five new volunteers with normal or corrected-to-normal vision served as subjects. They were naive concerning the purposes of the experiment.

The mean number of transparency judgments of the nine stimuli satisfying constraints $i$ through iv is 23.9, with a SD of 2.0. Stimulus acdb (stimulus 16) again evoked a significant number of transparency judgments. Fourteen subjects judged stimulus acdb as transparent. Only one other of the crisscross stimuli was seen as transparent. Three subjects judged stimulus bdca (stimulus 18) as transparent. We have suggested that it is the closeness in reflectance of regions $\mathrm{c}$ and $\mathrm{d}$ on stimulus acdb that is responsible for the relatively large number of transparency judgments. This is supported by the finding that transparency judgments varied inversely with the salience with which constraints $i$ and ii are violated. The number of transparency judgments with the stimulus order cdab were $17,10,5$, and 2 when the reflectance of $d$ exceeded that of $c$ by $1 \%, 2 \%, 4 \%$, and $8 \%$, respectively. The number of transparency judgments with the stimulus order cdba were 19 and 4 when the reflectance difference between $c$ and $d$ exceeded that between a and b by $4 \%$ and $27 \%$, respectively.

Supplementary Experiment 2. Constraints i and ii are not sufficient to uniquely determine the perception of transparency. For example, Table 1 shows that stimuli abdc, adbc, cbda, and cdba all satisfy the order and magnitude constraints. If surface $B$ is seen as overlying surface $D$, then stimulus abdc becomes adbc, and stimulus adbc becomes abdc. Similarly, stimulus cbda becomes cdba and stimulus cdba becomes cbda. Auxiliary principles become necessary to predict whether surface $D$ is seen as transparent and overlying surface B or surface B is seen as transparent and overlying surface $D$ when subjects are not instructed to see a particular arrangement, as in Experiment 1 . One possible principle is that region $\mathrm{c}$ in Figure 2 is joined to regions $b$ or $d$, depending on which it differs least from in lightness (Figures $4 \mathrm{~g}$ and 4h). An experiment was conducted to test this possibility. 
Table 3

Stimulus Reflectances and Frequency of Transparency Judgments in Supplementary Experiment 1

\begin{tabular}{|c|c|c|c|c|c|c|c|c|}
\hline \multirow[b]{2}{*}{ Stimulus No. } & \multirow[b]{2}{*}{ Stimulus } & \multicolumn{6}{|c|}{ Reflectances } & \multirow[b]{2}{*}{ Frequency } \\
\hline & & $\mathrm{a}$ & $b$ & $c$ & d & $a$ & $\mathrm{e}$ & \\
\hline \multicolumn{9}{|c|}{ Satisfied Constraints i and ii } \\
\hline 1. & abdc & .09 & .22 & .39 & .30 & .69 & .77 & 19 \\
\hline 2. & bcda & .54 & .27 & .46 & .49 & .11 & .48 & 25 \\
\hline 3. & bacd & .36 & .17 & .51 & .58 & .37 & .71 & 23 \\
\hline 4. & bcad & .40 & .15 & .39 & .52 & .52 & .65 & 25 \\
\hline 5. & bcda & .56 & .17 & .43 & .48 & .13 & .47 & 25 \\
\hline 6. & bcda & .31 & .08 & .12 & .23 & .48 & .16 & 25 \\
\hline 7. & adcb & .06 & .54 & .48 & .31 & .35 & .45 & 23 \\
\hline 8. & adcb & .10 & .37 & .29 & .16 & .48 & .22 & 25 \\
\hline 9. & adcb & .11 & .49 & .37 & .19 & .47 & .26 & 25 \\
\hline $\begin{array}{c}\text { Mean } \\
\text { SD }\end{array}$ & & & & & & & & $\begin{array}{r}23.9 \\
2.0\end{array}$ \\
\hline \multicolumn{9}{|c|}{ cdab Stimuli } \\
\hline 10. & cdab & .19 & .52 & .13 & .14 & -.03 & .14 & 17 \\
\hline 11. & $c d a b$ & .16 & .48 & .09 & .11 & -.06 & .11 & 10 \\
\hline 12. & cdab & .14 & .43 & .06 & .10 & -.14 & .10 & 5 \\
\hline 13. & cdab & .18 & .59 & .05 & .13 & -.20 & .14 & 2 \\
\hline $\begin{array}{c}\text { Mean } \\
\text { SD }\end{array}$ & & & & & & & & $\begin{array}{l}8.5 \\
6.6\end{array}$ \\
\hline \multicolumn{9}{|c|}{ cdba Stimuli } \\
\hline 14. & cdba & .52 & .49 & .40 & .47 & 2.33 & .56 & 19 \\
\hline 15. & cdba & .61 & .55 & .18 & .50 & 6.40 & .63 & 4 \\
\hline Mean & & & & & & & & 11.5 \\
\hline SD & & & & & & & & 10.6 \\
\hline \multicolumn{9}{|c|}{ Crisscross Stimuli } \\
\hline 16. & $\mathrm{acdb}$ & .27 & .44 & .36 & .37 & -.06 & .36 & 14 \\
\hline 17. & bdac & .34 & .24 & .41 & .31 & -1.00 & .33 & 0 \\
\hline 18. & bdca & .50 & .23 & .35 & .32 & -.11 & .34 & 3 \\
\hline 19. & $\mathrm{cadb}$ & .21 & .38 & .19 & .25 & -.35 & .24 & 0 \\
\hline Mean & & & & & & & & 4.3 \\
\hline SD & & & & & & & & 6.7 \\
\hline
\end{tabular}

Note-The values of $a$ and $e$ are given by Equations 3 and 4. A negative value for a indicates that a stimulus violates constraint $i$ and for $e$ that it violates constraint iii. An absolute value greater than 1 for a indicates that a stimulus violates constraint ii and for e that it violates constraint iv.

The six stimuli abdc, cdba, adbc, cbda, cadb, and dbac from Set 1 were presented individually, using four different random orders, to 40 subjects in two classes. To familiarize the subjects with the phenomenon, they were shown pictures of surfaces arranged as in configuration II, in which the perception of transparency both occurred and failed to occur. The subjects were asked to indicate on a data sheet, first, whether a stimulus was seen as transparent, and second, if a stimulus was seen as transparent, whether the top square was seen to overlie the bottom square or whether the bottom square was seen to overlie the top square. The subjects were instructed to base their judgments on their immediate visual impressions. To avoid position biases, 19 subjects were presented with the stimuli upright and 21 with the stimuli inverted.

Stimuli cadb and dbac have crisscrossing gray levels and should be seen as not transparent. For stimuli abdc and cdba, region $c$ is closer in lightness to region $d$ than to region $b$ whereas, for stimuli adbc and cbda, region $c$ is closer in lightness to region $b$ than to region d. According to the hypothesis proposed, with stimuli abdc and cdba, surface D should be seen to overlie surface $B(D / B)$ and, with stimuli adbc and cbda, surface $B$ should be seen to overlie surface $D(B / D)$.

Table 4 presents the number of nontransparent judgments, of $D / B$ transparent judgments, and of $B / D$ transparent judgments. The frequencies in Table 4 combine the jugments made with both the upright and inverted presentations of the stimuli. With stimuli cadb and dbac, the number of nontransparency judgments was 66 and that of transparency judgments was 14 . The reason for the larger number of transparency judgments here than in Experiment 1 is not clear. It may reflect a criterion difference. The instructions in Experiment 1 were given individually and emphasized that a stimulus was to be judged transparent only if the subject could see through the overlying surface to both underlying surfaces. For stimuli abdc and cdba, the total number of 
Table 4

Distribution of Transparency Judgments in Supplementary Experiment 2

\begin{tabular}{ccccc} 
& & \multicolumn{3}{c}{ Number of Judgments } \\
\cline { 3 - 5 } Stimulus & & Transparent & Transparent & Not \\
No. & Stimulus & D/B & B/D & Transparent \\
\hline
\end{tabular}

\begin{tabular}{|c|c|c|c|c|}
\hline \multicolumn{5}{|c|}{$c$ Closer to $d$ in Lightness } \\
\hline 1. & abdc & 32 & 4 & 4 \\
\hline 2. & cdba & 29 & 10 & 1 \\
\hline Means & & 30.5 & 7.0 & 2.5 \\
\hline SDs & & 2.1 & 4.2 & 2.1 \\
\hline \multicolumn{5}{|c|}{ c Closer to b in Lightness } \\
\hline 3. & adbc & 8 & 26 & 6 \\
\hline 4. & cbda & 12 & 28 & 0 \\
\hline Means & & 10.0 & 27.0 & 3.0 \\
\hline SDs & & 2.8 & 1.4 & 4.2 \\
\hline \multicolumn{5}{|c|}{ Crisscross Stimuli } \\
\hline 5. & $\mathrm{cadb}$ & 3 & 5 & 32 \\
\hline 6. & dbac & 1 & 5 & 34 \\
\hline Means & & 2.0 & 5.0 & 33.0 \\
\hline SDs & & 1.4 & 0.0 & 1.4 \\
\hline
\end{tabular}

Note $-D / B=$ Surface $D$ is seen to overlie surface $B ; B / D=$ Surface $B$ is seen to overlie surface $D$ (see Figure $2 a$ ).

transparency judgments of $\mathrm{D} / \mathrm{B}$ was 61 and that of B/D was 14. For stimuli adbc and cbda, the total number of transparency judgments of $B / D$ was 54 and that of $D / B$ was 20 . A t test tested the hypothesis that there is a presumption to unite regions having more similar lightnesses. The number of $B / D$ judgments were subtracted from the number of $D / B$ judgments. The mean of the difference scores for stimuli abdc and cdba was 23.5, and that for cbda and adbc, -17.0. A t test of the difference between the two means is significant $[\mathrm{t}(2)=8.79, \mathrm{p}<.05]$. The results also suggest a position bias. Twenty-two of the 34 judgments counter to the hypothesis were judgments that the top square was transparent and overlay the bottom square. The percentage of judgments differ significantly from the $50 \%$ change level at the .05 significance level $(z=1.88)$. The fact that, in Experiment 1, stimuli adbc and cbda gave as many transparency judgments of $\mathrm{D} / \mathrm{B}$ as did stimuli abdc and cdba indicates that the predisposition to unite regions that are closer in lightness can easily be overcome by an instructional set.

\section{EXPERIMENT 2}

Experiment 2 investigated how violations of constraints iii and iv affect the perception of transparency.

\section{Method}

Stimuli. Ten stimuli arranged as in configuration II were constructed from gray papers. Four papers differing in reflectance depicted a central rectangle overlying a bipartite background. The rectangle was $2 \times 1 \mathrm{~cm}$ and consisted of two adjacent squares $1 \mathrm{~cm}$ on a side. The background consisted of two adjacent squares $2.5 \mathrm{~cm}$ on a side. Table 5 lists the stimulus reflectances. Negative values of $e$ indicate that stimuli 1 and 3 violated constraint iii. Constraint iii is satisfied if the reflectance of $c$ in stimulus 1 is increased by $5 \%$ and in stimulus 3 by $8 \%$. Since the value of e is undefined, stimulus 7 also violated constraints iii and iv. Constraints iii and iv are satisfied if the reflectance of $c$ in stimulus 7 is increased by $13 \%$. The negative values for $a$ indicate that stimuli $2,4,6$, and 8 violated constraint $i$. The values of a greater than 1 indicate that stimuli 9 and 10 violated constraint ii. The values of $a$ and $e$ indicate that stimulus 5 satisfies constraints i through iv.

Procedure. The procedure was similar to that in Experiment 1. Transparency was first illustrated by showing subjects a Polaroid filter that could be rotated from clear to opaque. The subjects were then shown computer-generated pictures of surfaces arranged as in Figure 3b. The 10 stimuli were mounted on pieces of black cardboard and presented by means of a Gerbrands tachistoscope. The subjects were instructed to report whether the center regions $d$ and c were seen as transparent. They were told to judge a stimulus as transparent only if both background regions, $a$ and $b$, could be seen through regions $d$ and $c$, respectively. If only region a could be seen through region $d$ or only region $b$ through region $c$, then a subject was told to judge the stimulus as not transparent. The subjects were told to make their judgments based upon their immediate visual impressions. The exposure duration was $1.5 \mathrm{sec}$. Four computer-generated pictures of surfaces arranged as in configuration II were presented as practice stimuli. Two of the practice stimuli produced a perception of transparency and 2 did not. To familiarize a subject with the stimuli, the 10 stimuli were presented in a preliminary trial during which no judgments were made. The stimuli were presented in a different random order to each subject.

Subjects. Fifteen volunteers with normal or corrected-to-normal vision served as subjects. They were naive concerning the purpose of the experiment.

\section{Results}

Table 5 shows that, except for stimulus 10, the perception of transparency occurred infrequently when either constraint $i$ or constraint ii was violated. The reason for the six transparency judgments of stimulus 10 is not clear. In contrast, the perception of transparency occurred frequently with violations of either constraint iii or constraint iv. Stimulus 5, which satisfied constraints i through iv, was judged transparent by 13 subjects. Stimulus 1 , which violated constraint iii, was judged transparent by 14 subjects. Stimulus 3, which also violated constraint iii, was judged transparent by 10 subjects. Stimulus 3 fails to differ significantly from stimulus $5[t(14)=$ $1.38, \mathrm{p}>.05$ ] but differs significantly from stimulus $1[t(14)=2.26, p<.05]$. The smaller number of transparency judgments with stimulus 3 is due, we believe, to stimulus 3's failing to satisfy constraint ii in terms of lightness. Lightness is a nonlinear function of reflectance and the magnitude relation in constraint ii can be satisfied or violated independently by lightness and reflectance. Matching the lightnesses to Munsell values showed that the lightness difference between $a$ and $b$ on stimulus 1 equals the lightness difference between $c$ and $d$. On stimulus 3, the lightness difference between $c$ and $d$ is approximately .75 of a Munsell step greater than the lightness difference between $a$ and $b$. This is consistent with our suggestion that the important variable in satisfying constraint ii is lightness and not reflectance or luminance. Table 5 also shows that stim- 
Table 5

Stimulus Reflectances and Frequency of Transparency Judgments in Experiment 2

\begin{tabular}{|c|c|c|c|c|c|c|c|}
\hline \multirow[b]{2}{*}{ Stimuli } & \multicolumn{6}{|c|}{ Reflectances } & \multirow[b]{2}{*}{ Frequency } \\
\hline & $\mathbf{a}$ & $\mathrm{b}$ & $\mathrm{c}$ & d & $a$ & e & \\
\hline 1. cdba & .84 & .47 & .14 & .33 & .51 & -.21 & 14 \\
\hline 2. dcba & .84 & .47 & .33 & .14 & -.51 & .38 & 0 \\
\hline 3. cdba & .77 & .41 & .08 & .29 & .58 & -.38 & 10 \\
\hline 4. dcba & .77 & .41 & .29 & .08 & -.58 & .33 & 2 \\
\hline 5. cdba & .84 & .47 & .22 & .33 & .30 & .11 & 13 \\
\hline 6. dcba & .84 & .47 & .33 & .22 & -.30 & .36 & 1 \\
\hline 7. cdba & .92 & .66 & .22 & .48 & 1.00 & u & 14 \\
\hline 8. dcba & .92 & .66 & .48 & .22 & -1.00 & .57 & 2 \\
\hline 9. cbda & .66 & .49 & .05 & .53 & 2.82 & .73 & 2 \\
\hline 10. $\mathrm{adbc}$ & .30 & .53 & .92 & .40 & 2.26 & .22 & 6 \\
\hline
\end{tabular}

Note-The values of $a$ and $e$ are given by Equations 3 and 4 . A negative value for a indicates that a stimulus violates constraint $i$ and a negative value for $e$ that it violates constraint iii. An absolute value greater than 1 for a indicates that a stimulus violates constraint ii and for $e$ that it violates constraint iv. $u=e$ is undefined since $(a+c)-(b+d)$ equals 0.

ulus 7 , which violated both constraint iii and constraint iv, was judged transparent by 14 subjects. The results, therefore, indicate that violations of constraints iii and iv fail to affect the perception of transparency. ${ }^{3}$

\section{FILTER MODEL}

The following question may be raised: Since constraints $i$ and ii are not wholly ecologically representative, why do they predict the occurrence of transparency as well as they do? The luminances of the areas $d$ and $c$ in Figure 1 are the result of stimulation of the retina by the light reflected from the episcotister and from surfaces $A$ and $B$ behind the episcotister. The resulting addition of luminances is known as additive color mixture and is given quantitatively by Talbot's law. Additive color mixture occurs in some natural scenes as, for example, with clouds of dust. Many common occurrences of transparency, however, are in terms of subtractive rather than additive color mixtures. When an object is viewed through a liquid, mist, or glass, subtractive color mixture occurs. The luminance of the overlapping area in subtractive color mixture is the result of the light intensity reflected by the background surface and transmitted by the transparent medium plus the light intensity reflected by the transparent medium.

What are the relations among image intensities when transparency occurs in terms of subtractive color mixture? The physical situation is depicted in Figure 5a. We will assume an achromatic surface viewed in neutral illumination through a transparent medium that is nonselective for wavelength. In Figure $5 a, a$ is the reflectance of surface $A, b$ is the reflectance of surface $B, f$ is the reflectance of filter $F$, and $t$ is the transmittance of the filter. Figure $5 b$ illustrates the pattern of reflectance and transmittance assumed to occur. The apparent reflectances of regions $d$ and $c$ are equal to

$$
\begin{aligned}
& d=f+\left(t^{2} a\right) /(1-f a), \\
& c=f+\left(t^{2} b\right) /(1-f b) .
\end{aligned}
$$

Solving Equations 5 and 6 for $t$ and f yields

$$
\begin{gathered}
t=\sqrt{\frac{\left(C-b c d+b d^{2}-d\right)\left(b-a-a b c+a^{2} c\right)}{(b-a+a b d-a b c)^{2}}} \\
f=(b d-a c) / b(1+a d)-a(1+b c) .
\end{gathered}
$$

Order and magnitude constraints for the perception of transparency with subtractive color mixture can be derived from Equations 7 and 8 . Since the perception of transparency occurs when $t$ is restricted to values between 0 and 1, Equation 7 implies (v) (c-bcd + $\left.b^{2}-d\right)\left(b-a-a b c+a^{2} c\right)>0$ and (vi) $(b-a+a b d-$ $a b c)^{2}>\left(c-b c d+b d^{2}-d\right)\left(b-a-a b c+a^{2} c\right)$. Since the reflectance of the filter, $f$, is also restricted to values greater than or equal to 0 and less than 1 , Equation 8 implies (vii) if bd $>$ ac, then $b(1+a d)$ $>a(1+b c)$, and vice versa if $b d<a c$, and (viii) the absolute difference $|b(1+a d)-a(1+b c)|$ must be greater than the absolute difference $\mid$ bd - ac $\mid$. Constraints $v$ and vii insure that $t$ and $f$ are positive, and constraints vi and viii insure that $t$ and $f$ are less than 1. An additional constraint is that $t+f$ must be less than or equal to 1 .

What is the relationship between the equations derived from the episcotister and the filter models? Equations 1 and 2 are clearly not equal to Equations 5 and 6 . For example, if $a=.5, b=.3, t=.7$, and $e$ and $f=.2$, $d$ and $c$ are equal to .41 and .27 from Equations 1 and 2 and $d$ and $c$ are equal to .47 and .36 from Equations 5 and 6 . The order and magnitude constraints defining the boundary conditions for solutions of the two sets of equations, however, appear to be closely related. Equations 5 and 6 of the 


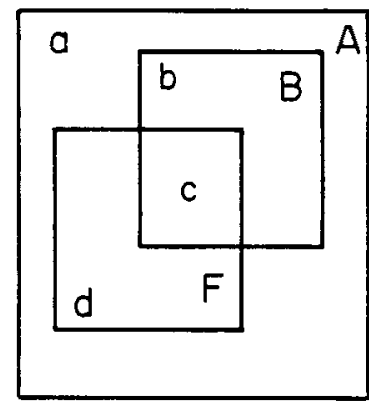

(a)

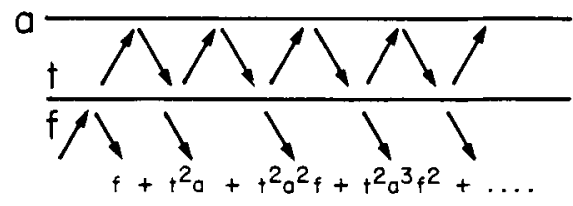

(b)

Figure 5. (a) Illustration of subtractive color mixture occurring with a filter. Capital letters A, B, and F Indicate the background surfaces and the filter. Lowercase letters indicate regions of differing apparent reflectance. (b) Ilustration of the pattern of reflectunce (see text).

filter model imply constraints $\mathrm{i}$ and $\mathrm{ii}$ derived from Equation 3 of the episcotister model, and Equations 1 and 2 of the episcotister model imply constraints $v$ and vi derived from Equation 7 of the filter model. ${ }^{4}$ Although we have not been able to demonstrate it mathematically, a computer search of the solutions to Equations 5 and 6 of the filter model has failed to find any solutions that violate constraints iii and iv derived from Equation 4. Similarly, a computer search of the solutions to Equations 1 and 2 of the episcotister model has failed to find any solutions that violate constraints vii and viii derived from Equation 8 of the filter model. The variables were incremented by .02 within the bounds for each set of equations, and the calculations were carried out to four decimal places. What is suggested is that the solution sets of Equations 1 and 2 and Equations 5 and 6 are the same, or very nearly the same.

The physical occurrences of transparency involve both color addition and color subtraction and set the normative conditions for the perception of transparency. If perception is to be adaptive, it must satisfy these conditions, except in unimportant ways. This does not mean, however, that the visual system solves Equations 1 and 2 and Equations 5 and 6 . To judge transparency, the visual system may utilize heuristics, which, in the main, agree with physical reality. Constraints i and ii derived from Metelli reflect order and difference relations that occur both with additive and subtractive color mixture. The two constraints are, therefore, ecologically valid indicators of physical transparency and can serve as a basis for adaptive behavior. The constraints with additive and subtractive color mixture are, of course, not always the same. In the case of hue, for example, yellow plus blue yields white with additive color mixture, whereas yellow plus blue yields green with subtractive color mixture. Beck $(1972,1975)$ presented demonstrations showing that hue transparency occurs with subtractive color mixture.

\section{EXPERIMENT 3}

We have indicated that the equations describing transparency with additive and subtractive color mixture are not quantitatively equal. Transparency judgments based on Equation 3, for example, will not be quantitatively correct with subtractive color mixture. This is, howerver, not very important, because in general one is not able to make quantitatively accurate judgments of transparency. Transparency estimates appear to be based not on luminance or reflectance values but on lightness values. Metelli's Equations 1 and 2 describing transparency assume that transparency is determined by the physical luminance or reflectance values. Equal increments of reflectance, however, do not represent equal increments of lightness. For example, the lightness difference between two papers that have reflectances of $80 \%$ and $90 \%$ is .45 of a Munsell step, whereas the difference between papers having reflectances of $5 \%$ and $15 \%$ is 1.82 Munsell steps. Since the visual system does not have direct information about reflectances, it is likely that transparency judgments will vary linearly, not with reflectance difference, but with lightness difference. Thus, to predict quantitative judgments of transparency, one must introduce a psychophysical function, such as the Munsell value scale, which describes how lightness varies as a function of reflectance. The aim of Experiment 3 was to determine whether transparency judgments varied linearly with reflectance difference or with lightness difference.

\section{Method}

The stimulus consisted of three intensity regions arranged to depict two overlapping rectangles, as shown in Figure 6a. The stimulus color of region a was a Munsell value of $1(1.2 \%$ reflectance), that of region b a Munsell value of $8(59.1 \%)$, and that of region $c$ a Munsell value of $4(12 \%)$. The stimulus is ambiguous and can be seen as the upright rectangle overlying the diagonal rectangle or of the diagonal rectangle overlying the upright rectangle. The rectangles were $2.6 \times 4.0 \mathrm{~cm}$.

The Munsell papers were pasted on a matte black background. The background was cut away so that only the stimulus figure was visible. The stimuli were supported by a stalk fitted into a wooden base located at eye level $5 \mathrm{ft}$ from a subject. The illumination came from a projector using a bulb with a color temperature of $2900 \mathrm{~K}$. The light passed through a 1-62 Corning filter which converts the 


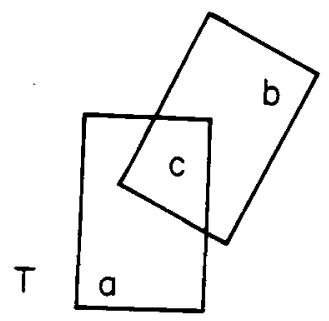

(a)

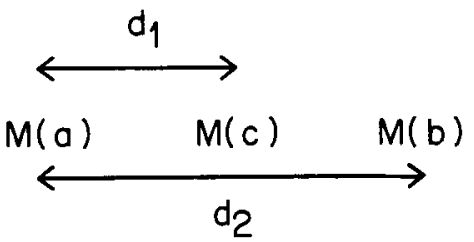

(b)

Figure 6. (a) Stimulus figure in Experiment 3. (b) Relative simllarity of region $c$ to that of regions a and $b$ (see text).

illuminant to C. I. E. illuminant C $(6500 \mathrm{~K})$. Subjects viewed the stimuli monocularly through a viewing tube that limited visibility to the immediate stimulus surround. An electrically controlled shutter limited viewing time to $2 \mathrm{sec}$. The subjects were allowed to view a stimulus for as many $2-\sec$ periods as they wished, but were urged to make their judgments upon their immediate visual impressions.

The subjects were first acquainted with the phenomenon of transparency through examples. The test stimulus was presented together with 10 other stimuli made up of colored Munsell papers. ${ }^{3}$ The subjects judged the chromatic stimuli only with respect to whether they were seen as transparent or not. Following presentation of the chromatic stimuli, the subjects were told that the next stimulus would be achromatic, and that they were to judge the stimulus not only with respect to whether it appeared transparent but also with respect to how transparent it appeared to be. They were told that a surface could vary in transparency. Differing degrees of transparency were described by asking subjects to think about mixing ink with water or milk with water. Adding milk or ink to the water decreases the transparency of the mixture. The subjects were asked to judge whether they saw transparency or not. If a subject reported seeing transparency, he or she was then asked whether the upright rectangle or the diagonal rectangle was seen as the overlying surface. The subjects were asked to estimate the transparency of the overlying surface from near $0 \%$ (nearly completely opaque) to near $100 \%$ (nearly completely transparent). Following the transparency judgment, the subjects were asked to try to see the three regions as coplanar and to match the lightness of the regions to an 18-step Munsell value scale. The Munsell scale was placed on a shelf to the left of a subject and illuminated by an Easel lamp which simulated C.I.E. illuminant $C$. The subjects were allowed to view the stimulus without time limit while matching the stimulus to the Munsell grays.

Subjects. Eleven subjects served in the experiment. They all had normal or corrected-to-normal vision, and were naive about the purpose of the experiment.

\section{Results and Discussion}

In Figure 6a, a represents the reflectance of the top surface, $T$, and $b$ the reflectance of the bottom surface, $B$. The transparency of the top surface in the area of overlap is represented by $a$. According to Talbot's law, the apparent reflectance of region $c$ is equal to

$$
c=\alpha b+(1-\alpha) a,
$$

where $\alpha$ and $(1-\alpha)$ represent, respectively, the proportions of the reflectances of surfaces $B$ and $T$ that make up the apparent reflectance c. Solving Equation 9 for $\alpha$, yields

$$
a=(c-a) /(b-a) .
$$

If the upright black surface is seen as transparent, the perceived transparency calculated in terms of reflectance is .19 and in terms of Munsell value, .43. The means of the subjects' lightness matches of regions a, $c$, and $b$ were Munsell values of 3.1, 5.5, and 9.2. The transparency estimate when these values are substituted in Equation 10 is .39. The mean of the subjects' transparency estimates is 41.4 with a standard deviation of 5.1. Thus, one might introduce into Equation 10 lightness values rather than reflectances to accurately predict quantitative transparency judgments.

The finding that the perception of transparency is determined not by the physical reflectances, but by lightness values, argues that the phenomenon of transparency is not based on Talbot's law. In fact, it one sets up an episcotister, judgments of transparency would be inaccurate. Rather, the perception of transparency appears to be based on the lightness values, which are a nonlinear function of reflectance. What is the theoretical justification for using the Munsell values of regions a, b, and $c$ in Equation 10? A justification may be given based on the hypothesis that the perceived transparency is a function of the relative similarity of the lightness of region $c$ to the lightness of the underlying region $b$ and to the lightness of the overlying region a. The perception of transparency is the result of encoding the lightness of region $c$ as the lightness of the underlying region $b$ modified by the lightness of the overlying region $a$. The more similar the lightness of $\mathrm{c}$ is to the lightness of $a$, the less the perceived transparency, and the more similar the lightness of $c$ is to the lightness of $b$, the greater the perceived transparency. The Munsell value scale is based on direct estimates of lightness differences and reflects lightness similarities. If $M(a)$, $M(b)$, and $M(c)$ are the Munsell values of the regions $a, b$, and $c$, the relative similarity of lightness $c$ to to lightness $a$ is given by the difference, $\mathrm{dl},[\mathrm{M}(\mathrm{c})-$ $M(a)$ ] divided by the difference, d2, [M(b)-M(a)] (Figure 6b). The equation for perceived transparency has the same form as Equation 10, but with Munsell values substituted for reflectances:

$$
a=M(c)-M(a) / M(b)-M(a) .
$$


Partial transparency. The perception of transparency with a three-part stimulus is anomalous. One does not perceive a transparent surface through which other objects and surfaces are seen. Rather, one perceives a surface that is in part transparent and in part opaque. Metelli (1974a) has called this special kind of transparency "partial transparency." As pointed out above, a model based on Talbot's law and color addition requires that the reflectance of the overlapping region, $c$, lie between the reflectance of the $a$ region and the reflectance of the $b$ region. The similarity algorithm we have proposed for judging the degree of transparency also requires that the lightness of the overlapping region, $c$, lie between the lightness of the a region and the lightness of the $b$ region. In contrast, a model based on a filter and subtractive color mixture allows the reflectance of the overlapping region to be greater than the reflectances of the nonoverlapping regions. This occurs when the reflectance of the top surface is greater than the reflectance of the bottom surface. Equation 6 gives the reflectance of the overlapping region, $c$, when $F$ and $B$ in Figure $5 a$ are interpreted to be the two overlapping surfaces. It is of interest to note that there is a physical interpretation with additive color mixture in which the intensity of the overlapping region is greater than that of the nonoverlapping regions. This occurs if B and T in Figure 6 are two projected rectangles of light. Partial transparency, as pointed out, is not ecologically representative and appears to occur because of a preference by the visual system for minimizing the complexity of the perceptual interpretation. The perception of transparency in Figure 7 both simplifies the shapes and minimizes the lightness changes. Figure 7 shows examples of a three-part stimulus in which the reflectance of the overlapping region is below and above that of the nonoverlapping regions. Nineteen of the 26 subjects judged the stimulus shown in Figure 7a to be transparent, but only 6 subjects judged the stimulus shown in Figure $7 \mathrm{~b}$ to be transparent. Thus, judgments of transparency do occur when the reflectance of the overlapping region fails to lie between the reflectances of the nonoverlapping regions.

\section{EXPERIMENT 4}

Metelli has called the perception of transparency "complete transparency" when a transparent film is perceived to overlie two opaque surfaces differing in reflectance. Equation 3 gives the degree of transparency in the Metelli model for complete transparency. We believe that, with complete transparency, as with partial transparency, the degree of perceived transparency will not be correctly predicted when physical reflectances are substituted in Equation 3. One possibility is that substituting lightness values for reflectances in Equation 3 correctly predicts the perceived
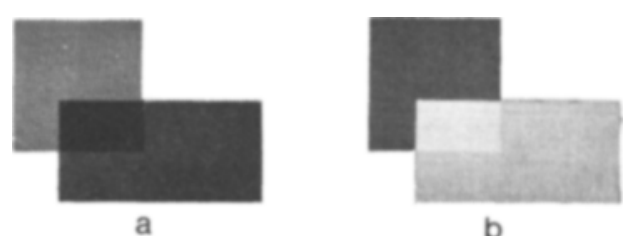

Figure 7. Three-part stimull violating the constraint that the lightneas of $c$ lie between the lightnesses of $b$ and $d$ (see text).

degree of transparency. The argument for this is that the estimate of transparency is based on the reduction of apparent contrast. The perception of the degree of transparency is assumed to be a function of the similarity of the lightnesses of regions $d$ and $c$ relative to the similarity of the lightnesses of regions $a$ and $b$ (Figure 2). If the lightnesses of regions $d$ and $c$ are equal, that is, if their contrast is zero, then the degree of perceived transparency is zero. As the lightness difference between regions $d$ and $c$ approaches the lightness difference between regions $a$ and $b$, the perceived degree of transparency goes to $100 \%$. This equation, however, cannot be correct without further restriction. Consider stimulus 9 in Table 6. The lightness difference between $d$ and $c$ is nearly equal to that between $a$ and $b$. Subjects' mean lightness match of region a was a Munsell value (MV) of 7.8 , b was a MV of 6.5, c a MV of 2.7, and d a MV of 3.9. Stimulus 9 was arranged as in configuration $I$ and could be seen as either surface D overlying surface $B$ or as surface B overlying surface D. Substituting lightness values in Equation 3 gives transparencies of .96 when $D$ is seen to overlie B and .99 when B is seen to overlie D. Transparencies of .96 and .99 imply that the lightness of region $d$ is close to that of region a and the lightness of region $c$ is close to that of region $b$. This is clearly not the case. Table 7 shows that the mean judged transparency was .38 when surface $D$ was seen to be overlying surface $B$ and .59 when surface $B$ was seen to be overlying surface $D$. The reason the formula is incorrect is that although the lightness difference $d-c$ is nearly equal to the lightness difference $a-b$, the lightnesses of $d$ and $a$ and of $c$ and $b$ are not close to each other. It is not clear what formula relates the perceived degree of transparency to lightness values in the case of complete transparency. The main aims of Experiment 4 were to determine (1) whether subjects are able to make reliable judgments of transparency in the case of complete transparency, and (2) whether a formula can be developed in terms of lightness values that will predict the perceived degree of transparency.

\section{Method}

Stimuli. Twenty-eight computer-generated stimuli were mounted on white cards. Six stimuli strongly violated constraints $i$ and ii and, in pretesting, were judged as not transparent. These served as catch stimuli. Seventeen stimuli were arranged as in con- 
Table 6

Stimulus Reflectances in Experiment 4

\begin{tabular}{|c|c|c|c|c|c|c|c|c|c|}
\hline & & \multicolumn{4}{|c|}{ Reflectances } & \multicolumn{2}{|c|}{$\mathrm{D} / \mathrm{B}$} & \multicolumn{2}{|c|}{$\mathrm{B} / \mathrm{D}$} \\
\hline \multicolumn{2}{|c|}{ Stimuli } & $\mathrm{a}$ & b & $\mathrm{c}$ & $\mathrm{d}$ & $a$ & e & $a$ & $\mathrm{e}$ \\
\hline 1. & bcda & .58 & .22 & .35 & .46 & .31 & .41 & -1.08 & .41 \\
\hline & $a d c b$ & .05 & .52 & .46 & .26 & .43 & .42 & -.29 & .42 \\
\hline 3. & bcad & .53 & .31 & .51 & .59 & .36 & .62 & 3.33 & .62 \\
\hline 4. & bcda & .56 & .21 & .35 & .48 & .37 & .43 & -1.75 & .43 \\
\hline 5. & dcba & .49 & .37 & .28 & .23 & -.42 & .31 & .35 & .31 \\
\hline & abcd & .09 & .21 & .40 & .43 & -.25 & .36 & .56 & .36 \\
\hline 7. II. & dcab & .23 & .38 & .18 & .13 & .88 & .08 & & \\
\hline 8. II. & bcda & .52 & .18 & .33 & .46 & .38 & .42 & & \\
\hline 9. & cdba & .46 & .31 & .07 & .14 & .47 & -.14 & .75 & -.14 \\
\hline 10. & cdba & .56 & .45 & .30 & .39 & .82 & -.38 & .88 & -.38 \\
\hline 11. & cbda & .50 & .24 & .14 & .32 & .69 & -.09 & .56 & -.09 \\
\hline 12. II. & cdba & .54 & .42 & .21 & .29 & .67 & -.21 & & \\
\hline 13. II. & cbda & .56 & .40 & .30 & .43 & .81 & -.13 & & \\
\hline 14. & $a d b c$ & .24 & .51 & .62 & .36 & .96 & 3.48 & .92 & 3.48 \\
\hline 15. II. & abdc & .10 & .26 & .59 & .45 & .88 & 2.90 & & \\
\hline 16. & cdba & .57 & .47 & .24 & .33 & .90 & -1.83 & .96 & -1.83 \\
\hline 17. & cbda & .62 & .36 & .23 & .49 & 1.00 & $\mathrm{u}$ & 1.00 & $\mathbf{u}$ \\
\hline 18. & abdc & .22 & .35 & .61 & .48 & 1.00 & $\mathbf{u}$ & 1.00 & $\mathrm{u}$ \\
\hline 19. & dcab & .43 & .65 & .38 & .23 & .68 & -.20 & 1.35 & -.20 \\
\hline 20. & bacd & .39 & .29 & .52 & .68 & 1.60 & -.09 & .79 & -.09 \\
\hline 21 . & abdc & .06 & .17 & .62 & .50 & 1.09 & -4.78 & 1.02 & -4.78 \\
\hline 22 & $a b d c$ & .06 & .16 & .57 & .46 & 1.10 & -3.94 & 1.03 & -3.94 \\
\hline
\end{tabular}

Note-The values of a are given by Equations 3 and 4 . A negative value for a indicates that a stimulus violates constraint $i$ and one for $e$ that it violates constraint iii. An absolute value greater than 1 for a indicates that a stimulus violates constraint ii and one for $e$ that it violates constraint iv. Roman numeral II following Stimulus number indicates that the stimulis arrangement is that of configuration $I I, u=e$ is undefined since $(a+c)-(b+d)$ equals $0 . D / B=$ Surface $D$ is seen to overlie surface $B: B / D=$ Surface $B$ is seen to overlie surface $D$ (see Figure 2a).

figuration I (Figures $2 a$ and $3 a)$ and five stimuli were arranged as in configuration II (Figure $3 \mathrm{~b}$ ).

Procedure. Two independent groups of subjects made stimulus judgments. One group of 10 subjects matched the lightness composing a stimulus to a chart of Munsell grays. The second group judged whether a stimulus appeared transparent and, if transparent, the degree of transparency.

In the lightness matching task, subjects were asked to match the lightnesses of the different regions composing a stimulus with the lightnesses on a Munsell chart ranging from 1 to 9.5 in .5 steps. The subjects were told that none of the Munsell values might seem like a perfect match, but that they should select the gray that appeared to be the best match. The subjects were first given a sample stimulus not used in the experiment and asked to match the lightnesses of the regions to the Munsell values. The subjects proceeded through the 22 noncatch stimuli at their own pace. The stimuli were presented using two different random orders.

In the transparency estimation task, the instructions were similar to those with Set 2 in Experiment 1 with the following differences. For configuration I stimuli, the subjects were first asked whether the overlying surface appeared transparent. If they said that the overlying surface did not appear transparent, the trial was concluded. If they reported the overlying surface to be transparent, they were then asked whether the rectangle $D$ was seen to overlie the square B (D/B), or whether the square B was seen to overlie the rectangle $D(B / D)$ (Figure 3a). For configuration II stimuli, the center square is always seen to overlie the background square. Subjects were, therefore, only asked to judge whether the center square appeared to be transparent (Figure $3 \mathrm{~b}$ ). The criteria for judging a stimulus as transparent were the same as with Set 2 in Experiment 1. As in Experiment 3, differing degrees of transparency were explained by asking subjects to think of mixing ink with water or milk with water. A visual example of the continuum from transparency to opacity was demonstrated by rotating crossed Polaroid filters from clear to opaque. The subjects estimated the degree of transparency on a scale of 0 to 100 .
The stimuli were exposed for $5 \mathrm{sec}$ in a Gerbrands tachistoscope. A subject initiated a trial by pressing a switch; he or she was allowed to view a stimulus for as many times as he or she wished. Before beginning the experiment, 10 practice stimuli were presented. Six of the practice stimuli were arranged as in configuration $I$ and four of them as in configuration II. The 28 stimuli were presented randomly. Two different random orders were used.

Subjects. Ten volunteers with normal or corrected-to-normal vision served as subjects in the lightness experiment. Twenty-six different volunteers with normal or corrected-to-normal vision served as subjects in the transparency experiment. ${ }^{\circ}$ All subjects were naive as to the purposes of the experiment.

\section{Results}

Table 6 lists the 22 noncatch stimuli, their reflectances, and the values of $\alpha$ and $e$ when a stimulus was seen as $\mathrm{D} / \mathrm{B}$ and when a stimulus was seen as $\mathrm{B} / \mathrm{D}$. Table 7 presents the number of transparency judgments of each stimulus, and the mean transparency estimates and their standard deviations. A Roman numeral II following a stimulus number indicates that the stimulus arrangement was that of configuration II.

Stimuli 1 through 8 in Table 6 satisfied constraints i through iv. Six were with configuration I and two with configuration II. Four of the configuration I stimuli (stimuli 1 through 4) satisfied the constraints when a stimulus was seen as D/B, and two (stimuli 5 and 6) did so when a stimulus was seen as $B / D$. The configuration II stimuli (stimuli 7 and 8 ) were always seen with the center square overlying the background 
Table 7

Frequency and Mean Transparency Estimates in Experiment 4

\begin{tabular}{|c|c|c|c|c|c|c|c|c|}
\hline & & \multicolumn{3}{|c|}{$\mathrm{D} / \mathrm{B}$} & \multicolumn{3}{|c|}{$B / D$} & \multirow{2}{*}{$\begin{array}{c}\text { Not } \\
\text { Transparent }\end{array}$} \\
\hline \multicolumn{2}{|c|}{ Stimuli } & Frequency & Mean & SD & Frequency & Mean & SD & \\
\hline 1. & bcda & 26 & .55 & .13 & 0 & & & 0 \\
\hline 2. & adcb & 26 & .59 & .16 & 0 & & & 0 \\
\hline & bcad & 25 & .49 & .22 & 1 & .25 & & 0 \\
\hline & bcda & 26 & .63 & .19 & 0 & & & 0 \\
\hline & dcba & 4 & .40 & .27 & 21 & .45 & .14 & 1 \\
\hline 6. & abcd & 2 & .25 & .21 & 24 & .64 & .20 & 0 \\
\hline 7. II. & dcab & 20 & .41 & .19 & 0 & & & 6 \\
\hline 8. II. & bcda & 22 & .53 & .20 & 0 & & & 4 \\
\hline & cdba & 12 & .38 & .27 & 9 & .59 & .28 & 5 \\
\hline & cdba & 8 & .67 & .16 & 15 & .60 & .22 & 3 \\
\hline 11. & cbda & 18 & .56 & .21 & 7 & .49 & .23 & 1 \\
\hline 12. II. & cdba & 23 & .43 & .20 & 0 & & & 3 \\
\hline 13. II. & cbda & 23 & .55 & .19 & 0 & & & 3 \\
\hline 14. & $\mathrm{adbc}$ & 17 & .66 & .18 & 5 & .35 & .24 & 4 \\
\hline 15. II. & abdc & 20 & .35 & .18 & 0 & & & 6 \\
\hline 16. & cdba & 16 & .46 & .20 & 7 & .57 & .24 & 3 \\
\hline 17. & cbda & 9 & .74 & .13 & 15 & .44 & .25 & 2 \\
\hline 18. & $\mathrm{abdc}$ & 12 & .45 & .25 & 5 & .69 & .09 & 9 \\
\hline 19. & $\mathrm{dcab}$ & 21 & .57 & .17 & 4 & .55 & .31 & 1 \\
\hline 20. & bacd & 13 & .69 & .13 & 13 & .59 & .19 & 0 \\
\hline 21 . & abdc & 20 & .38 & .18 & 2 & .88 & .11 & 4 \\
\hline 22. & $\mathrm{abdc}$ & 13 & .42 & .21 & 4 & .65 & .26 & 9 \\
\hline
\end{tabular}

Note $-D / B=$ Surface $D$ is seen to overlie surface $B ; B / D=$ Surface $B$ is seen to overlie surface $D$ (see Figure $2 a$ ). II=Configuration $I I$ (see Figure $3 b$ ).

square and are listed under $\mathrm{D} / \mathrm{B}$ in Table 6. The mean number of transparency judgments of these eight stimuli is 23.8 , with a SD of 2.4. Stimuli violating constraints iii and iv were also judged as transparent. The value of e remains the same and does not depend on whether a stimulus was seen as $D / B$ or B/D. Stimuli 9 through 13 violated only constraint iii, and stimuli 14 and 15 violated only constraint iv. Stimuli 16 through 18 violated both constraint iii and constraint iv. The mean number of transparency judgments of the 10 stimuli violating either constraint iii or constraint iv is 22.1 , with a SD of 2.3 .

Comparisons of stimuli that violated constraints only as D/B or B/D need to be made with care. Other factors, such as the violations of other constraints and the proximity of $c$ in lightness to $d$ or to $b$ may affect judgments. As in earlier experiments, Tables 6 and 7 show that violations of constraints $i$ and ii decreased the number of transparency judgments. Three stimuli violated only constraint $i$. Stimuli 5 and 6 violated constraint $i$ with the arrangement $D / B$, and stimulus 2 violated constraint $i$ with the arrangement $B / D$. The mean number of transparency judgments is 2.0, with a SD of 2.0. Stimulus 3 violated only constraint ii. This occurred with the arrangement $B / D$. The number of transparency judgments is 1.

Table 7 shows that subjects were able to make relatively consistent estimates of transparency. Correlation coefficients were computed between the predicted and obtained transparency estimates for stim- uli in which five or more subjects judged a stimulus as transparent. The correlation of the obtained transparency estimates with those predicted by Equation 3 is .62 for the eight stimuli satisfying constraints $i$ through iv. The hypothesis that transparency varies directly with $\alpha$ requires the intercept to be 0 and the slope to be 1 . The intercept of the regression equation is .28 and the slope, .66 . In addition to the 10 stimuli noted above which violated only constraints iii and iv, stimulus 19 violated constraint iii only when seen as $\mathrm{D} / \mathrm{B}$ and stimulus 20 violated constraint iii only when seen as $B / D$. The correlation between the predicted and obtained transparency estimates $(n=19$ when $D / B$ and $B / D$ are counted separately) is .28 . The intercept of the regression equation is .36 and the slope is .21. Thus, the results show that a fails to predict the degree of perceived transparency.

The stimulus relations underlying the perception of transparency may be quite different from the criteria used to judge the degree of transparency. We hypothesized that transparency depends on central visual processes checking whether the lightnesses in a pattern satisfy the order restrictions of constraint $i$ and the magnitude restrictions of constraint ii. Judgments of the degree of transparency may be based on other, not necessarily even consistent, stimulus relations. For configuration I stimuli, it is possible that subjects attended to the lightness relations among regions $b, c$, and $d$ in making transparency estimates. ${ }^{7}$ The estimation of transparency would then be predicted by an equation similar to Equation 11. An 
equation analogous to Equation 11 is given by Equation 12

$$
a=\frac{|c-d|}{|c-d|+|c-b|}
$$

The equation assumes that surface $D$ is seen to overlie surface $B$. If surface $B$ is seen to overlie surface $D$, then the numerator is $|c-b|$. The rationale for this equation is: (1) If $c$ equals $d$ in lightness, then transparency is $0 ;(2)$ the greater the difference in lightness between $c$ and $d$, the greater is the perceived transparency; and (3) the degree of perceived transparency is normalized so that it lies between 0 and 1 by dividing the absolute difference $|\mathrm{c}-\mathrm{d}|$ by the absolute difference $|c-d|+|c-b|$. If the lightness of $c$ is between the lightnesses of $b$ and $d$, Equation 12 reduces to Equation 11. The correlation for stimuli in which five or more subjects judged a stimulus as transparent $(n=25)$ between predicted and obtained transparency is .67 when mean lightness values are substituted in Equation 12 and .69 when reflectances are substituted. The slopes are .39 and .37 and the intercept values .37 and .36 , respectively. The results show that we do not as yet have a good understanding of the factors controlling the judgment of transparency with complete transparency.

\section{GENERAL DISCUSSION}

Perceptual transparency is a function of the stimulus information indicating that the overlying surface is not opaque, and it transmits, as well as reflects, light. Transparency is indicated by the alteration in image intensities produced by the overlying surface, the image distortions occurring because of light refraction, and the cues provided by figural configuration, depth, and motion. The finding in Experiment 3 that the perception of transparency is a function of lightness indicates that transparency is not, as suggested by Metelli (1974a, 1974b), the result of splitting a stimulus luminance into the luminance of the background surface and the luminance of the transparent surface, that is, the reverse of color fusion. In fact, constraints iii and iv which are based on the physical variables of reflectance or luminance appear not to be implemented by the visual system. The visual system appears only to be able to make order and relative distance judgments of lightnesses.

How do the cues deriving from the pattern of intensities relate to other stimulus information affecting the perception of transparency? The other variable that has been systematically studied is figural configuration. Metelli (1974b) has identified three main figural conditions for perceiving transparency: figural unity of the transparent layer, continuity of the boundaries in the transparent region with the boundaries of the nontransparent regions, and strat- ification of the transparent region into two overlapping layers. Our observations suggest that figural cues are primary and that if the figural configuration indicates the possibility of transparency, then the pattern of lightness relationships are checked to see if they are consistent with transparency. Transparency will be seen if the pattern of lightnesses satisfies constraints $i$ and ii. If more than one stratification of the surfaces satisfies the constraints, then instructional sets or subsidiary principles determine what is seen. One such principle is that the overlapping region tends to be seen as the continuation of the region from which it deviates least in lightness.

If the figural cues for transparency are strong enough, then transparency may be seen even when the pattern of lightness relationships is incorrect. A striking example of this is described by Metzger (1955). Metzger showed that if a disk is made to rotate slowly about a point, two intersecting circles on the disk will become separated in depth. There is a splitting of the intersecting region into two planes based on two kinds of contour movements. One plane is defined by contours that move into each other, and the other plane is defined by contours that are displaced over the retina. An observer sees a moving circle rotating around a stationary circle. What is of interest is that the cues for seeing overlapping circles ar so strong that the perception of transparency occurs for color combinations that strikingly violate both additive and subtractive color mixture. The present study shows that transparency can also occur with stationary stimulus patterns when the pattern of lightnesses are incorrect. For example, the stimulus order cdab with configuration I evoked judgments of transparency despite the violation of the order constraint by the pattern of lightnesses. When transparency occurs in such cases, the hues and lightnesses of the overlapping region may not appear correct. One needs to distinguish between the perception of transparency and color scissioning (we use "color scissioning" to refer to perceiving the color in the intersecting area as being composed of the base color and the overlying transparent color). Transparency can occur with and without color scissioning.

When does color scissioning occur? Metelli (1974a) explains color scissioning as a splitting of the stimulus luminance into the luminances of the background surface and of the overlying transparent surface. We have argued that color scissioning is not the reverse of Talbot's law of color fusion. Rather, it is a higher order, more cognitive encoding of the structural information in a stimulus. We hypothesize that color scissioning is the result of an encoding of a stimulus in terms of the color of an opaque surface and the color of a transparent surface overlying the opaque surface. Encoding in terms of a perceptual schema such as overlying planes of colors appears to 
require sensory support. Sensory support can occur in various ways. Phenomenological observation suggests that all colors can be described using the color names "red," "yellow," "green," "blue," "black," and "white." For example, orange can be described as a combination of a red color and a yellow color. Color scissioning can therefore occur in which an orange stimulus color is seen as a red through a yellow or vice versa. Another way of providing sensory support may be in terms of contrast colors at the boundaries of the transparent and the nontransparent regions, or flecks of the nontransparent color may leak through the transparent medium. Transparency with color scissioning occurs when sensory support leads to the visual system encoding the overlapping color as the opaque underlying color modified by the color of the transparent surface. When such an encoding is not induced in the visual system, the perception of transparency occurs without color scissioning, that is, one has impression of transparency, but the colors in the overlapping region are wrong.

\section{REFERENCES}

BECK, J. (1972). Surface color perception. Ithaca: Cornell University Press.

BECK, J. (1975). Surface color. Scientific American, 232(2), 62-75.

Bhill, M. (1976). Physical foundations of the perception of achromatic translucency. (Research Laboratory for Electronics Progress Reports, PR No. 117, pp. 315-320). Cambridge: M.I.T.

Metelli, F. (1974a). Achromatic color conditions in the perception of transparency. In R. B. MacLeod \& H. L. Pick (Eds.), Perception: Essays in honor of J. J. Gibson (pp. 95-116). Ithaca: Cornell University Press.

MetelLi, F. (1974b). The perception of transparency. Scientific American, 230(4), 90-98.

MetElli, F. (1975). Shadows without penumbra. In S. Ertel, L. Kemmler, \& L. Stadler (Eds.), Gestalttheorie in der modernen Psychologie (pp. 200-209). Darmstadt: Dietrich Steinkopff.
Metzger, W. (1955). Über Durchsichtigkeitserscheinungen. Rivista di Psicologia, Fascicolo Giubilare, 187-189.

\section{NOTES}

1. Metelli (1975) suggests the possibility of seeing a surface through a black transparent color when $e=0$. The density of the transparent color varies inversely with $a$.

2. Edge contrast increased the lightness difference between regions $c$ and $d$. A $1 \%$ difference in reflectance corresponds to a lightness difference of .08 of a Munsell step. When the stimulus was matched so that only regions $c$ and $d$ were visible, the lightnesses of the two regions were nearly indistinguishable. When viewed normally, the lightness difference between regions $c$ and $d$ was between .25 and .5 of a Munsell step.

3. It should be pointed out that the value of e can change greatly with small changes in reflectance. For example, if $a=.15, b=.28$. $c=.47$, and $d=.35, e=2.75$. If $c$ is decreased to .45 and $d$ increased to $.36, e=.83$. This makes it further unlikely that the visual system takes into account the value of $e$.

4. We are indebted to Seymour Haber of the National Bureau of Standards for these proofs. For a related mathematical treatment see Brill (1976).

5. The results with the chromatic stimuli will not be reported in this paper. In general, they showed that the perception of transparency occurs as readily with subtractive color mixture as with additive color mixture.

6. Twenty-nine subjects were run. One subject was dropped because he consistently called the catch stimuli transparent. Two subjects were dropped because they misunderstood the instructions. They thought that a stimulus to be reported transparent had to be seen as transparent when surface $D$ was seen to be overlying surface $B$ and when surface $B$ was seen to be overlying surface $D$.

7. There are two possibilities if a subject's transparency estimates are based on attending to three of the lightness values in a stimulus. A subject may attend to the lightness values of regions $b$, $c$, and $d$ or to the lightness values of regions $c, d$, and $a$. For configuration I stimuli, the overall pattern makes it seem likely that subjects would attend to regions b, $c$, and d. For configuration II stimuli, the two alternatives are equally likely. We decided, therefore, to test the hypothesis with configuration I stimuli.

(Manuscript received November 28, 1983; revision accepted for publication February 24, 1984.) 